Bull. Korean Math. Soc. 50 (2013), No. 4, pp. 1261-1275

http://dx.doi.org/10.4134/BKMS.2013.50.4.1261

\title{
CURVATURE IDENTITIES DERIVED FROM AN INTEGRAL FORMULA FOR THE FIRST CHERN NUMBER
}

\author{
Jungchan Lee, JeongHyeong Park, And Kouei Sekigawa
}

\begin{abstract}
We establish an integral formula for the first Chern number of a compact almost Hermitian surface and derive curvature identities from the integral formula. Further, we provide some results as applications of the identities.
\end{abstract}

\section{Introduction}

In [1], Berger derived a curvature identity on a 4-dimensional compact oriented Riemannian manifold from the generalized Gauss-Bonnet formula based on the fundamental fact that the Euler number is a topological invariant. Kuz'mina [11] and subsequently Labbi [12] extended Berger's result to any even-dimensional Riemannian manifold. Especially, Labbi showed that the obtained curvature identities hold without the compactness assumption. Euh, Park and Sekigawa [3] gave a direct proof for Labbi's result in the 4-dimensional case and some applications of the curvature identity $[4,5]$. We refer $[6]$ for the universality of the curvature identities in the Riemannian setting and we refer [7] for the universality of the curvature identities in the pseudo-Riemannain setting.

Motivated by the above observation, it is also worthwhile to study similar topics for the Riemannian manifolds equipped with some additional geometric structures such as almost complex structure, almost contact structure and so on. In this paper, we will focus on the following question:

Question A. What can we deduce from the integral formula for the Chern number of compact almost Hermitian surfaces?

We first establish an integral formula for the Chern number of a compact almost Hermitian surface. We regard the obtained integral formula as a functional on the space of all almost Hermitian structures on a compact complex

Received August 27, 2012.

2010 Mathematics Subject Classification. 53B20, 53C20.

Key words and phrases. Euh-Park-Sekigawa identity, first Chern number, almost Hermitian surface.

This work was supported by the National Research Foundation of Korea (NRF) grant funded by the Korea government (MEST) (2012-0005282). 
surface and derive a required curvature identity as the Euler-Lagrange equation of the integral based on Wu's Theorem [18]. In the last section, we provide some application of the curvature identity.

\section{Preliminaries}

In this section, we prepare some fundamental terminologies and formulas in almost Hermitian geometry which we need in our forthcoming discussions.

Let $M=(M, g, J)$ be a $2 n$-dimensional almost Hermitian manifold with the almost Hermitian structure $(g, J)$. In particular, we call a 4-dimensional almost Hermitian manifold an almost Hermitian surface briefly. We denote by $\omega$ the Kähler form of almost Hermitian structure $(g, J)$ defined by $\omega(X, Y)=$ $g(X, J Y)$, for $X, Y \in \mathfrak{X}(M), \mathfrak{X}(M)$ denoting the Lie algebra of all smooth vector fields on $M$. We assume that $M$ is oriented by the volume form $d v_{g}=$ $\frac{(-1)^{n}}{n !} \omega^{n}$. We denote the Riemannian connection, the curvature tensor, the Ricci tensor and the scalar curvature of $M$ by $\nabla, R, \rho$ and $\tau$, respectively. The curvature tensor $R$ is defined by

$$
R(X, Y) Z=\left[\nabla_{X}, \nabla_{Y}\right] Z-\nabla_{[X, Y]} Z
$$

for $X, Y, Z \in \mathfrak{X}(M)$. The Ricci $*$-tensor $\rho^{*}$ is a $(0,2)$-tensor field on $M$ defined by

$$
\begin{aligned}
\rho^{*}(X, Y) & =\operatorname{tr}(Z \mapsto R(X, J Z) J Y) \\
& =\frac{1}{2} \operatorname{tr}(Z \mapsto R(X, J Y) J Z)
\end{aligned}
$$

for $X, Y, Z \in \mathfrak{X}(M)$. The $*$-scalar curvature $\tau^{*}$ is the trace of the linear endomorphism $Q^{*}$ defined by $g\left(Q^{*} X, Y\right)=\rho^{*}(X, Y)$ for $X, Y \in \mathfrak{X}(M)$. The Ricci $*$-tensor $\rho^{*}$ satisfies

$$
\rho^{*}(X, Y)=\rho^{*}(J Y, J X)
$$

for any $X, Y \in \mathfrak{X}(M)$ [17]. Thus, $\rho^{*}$ is symmetric if and only if $\rho^{*}$ is $J$ invariant. By the definition $(2.2)$ of the Ricci $*$-tensor $\rho^{*}$, we can check that the Ricci $*$-tensor $\rho^{*}$ coincides with the Ricci tensor $\rho$ (and hence, also $\tau^{*}=\tau$ ) if $M$ is a Kähler. In this paper, we adopt the usual notational convention in the tensor analysis. For any local basis $\left\{e_{i}\right\}=\left\{e_{1}, e_{2}, \ldots, e_{2 n}\right\}$, we set

$$
\begin{aligned}
& g_{i j}=g\left(e_{i}, e_{j}\right), \quad J e_{j}=J_{j}{ }^{i} e_{i}, \quad R\left(e_{i}, e_{j}\right) e_{k}=R_{i j k}{ }^{l} e_{l}, \\
& \rho_{i j}=\rho\left(e_{i}, e_{j}\right), \quad \rho_{i j}^{*}=\rho^{*}\left(e_{i}, e_{j}\right), \quad\left(\nabla_{e_{i}} J\right) e_{j}=\nabla_{i} J_{j}{ }^{k} e_{k}, \\
& R_{i j k l}=g\left(R\left(e_{i}, e_{j}\right) e_{k}, e_{l}\right), \quad R\left(J e_{i}, e_{j}\right) e_{k}=R_{\overline{i j k}}{ }^{l} e_{l}, \\
& R_{\overline{i j k l}}=J_{i}{ }^{a} R_{a j k l}=g\left(R\left(J e_{i}, e_{j}\right) e_{k}, e_{l}\right), \quad R_{\bar{i} \bar{k} \bar{k} \bar{l}}=J_{i}{ }^{a} J_{j}{ }^{b} J_{k}{ }^{c} J_{l}{ }^{d} R_{a b c d}, \\
& \rho_{\bar{i} j}=J_{i}{ }^{a} \rho_{a j}=\rho\left(J e_{i}, e_{j}\right), \ldots, \rho_{\bar{i} \bar{j}}=J_{i}{ }^{a} J_{j}{ }^{b} \rho_{a b}=\rho\left(J e_{i}, J e_{j}\right), \\
& \rho^{*}{ }_{\overline{i j}}=J_{i}{ }^{a} \rho^{*}{ }_{a j}=\rho^{*}\left(J e_{i}, e_{j}\right), \ldots, \rho^{*}{ }_{\bar{i} \bar{j}}=J_{i}{ }^{a} J_{j}{ }^{b} \rho^{*}{ }_{a b}=\rho^{*}\left(J e_{i}, J e_{j}\right),
\end{aligned}
$$




$$
\begin{aligned}
& \nabla_{i} J_{j k}=g\left(\left(\nabla_{e_{i}} J\right) e_{j}, e_{k}\right)=g_{k l} \nabla_{i} J_{j}{ }^{l}, \\
& \nabla_{\bar{i}} J_{j k}=J_{i}{ }^{a} \nabla_{a} J_{j k}=g\left(\left(\nabla_{J e_{i}} J\right) e_{j}, e_{k}\right), \ldots, \nabla_{\bar{i}} J_{\bar{j} \bar{k}}=J_{i}{ }^{a} J_{j}{ }^{b} J_{k}{ }^{c} \nabla_{a} J_{b c},
\end{aligned}
$$

and so on. We also denote by $\left(g^{i j}\right)$ the inverse matrix of $\left(g_{i j}\right)$. Then, we have

$$
J_{i j}=-J_{j i}, \quad \omega_{i j}=-J_{i j}, \quad \nabla_{i} J_{j k}=-\nabla_{i} J_{k j}, \quad \nabla_{i} J_{\bar{j} \bar{k}}=-\nabla_{i} J_{j k} .
$$

Now, we denote real vector bundle of 2 -forms over $M$ by $\Lambda^{2} M$. Taking account of (2.4), we see that the vector bundle $\Lambda^{2} M$ can be decomposed as follows:

$$
\Lambda^{2} M=\mathbb{R} \omega \oplus \Lambda_{0}^{1,1} M \oplus L M \quad \text { (orthogonal direct sum), }
$$

where $\Lambda_{0}^{1,1} M$ denotes the vector bundle of real primitive $J$-invariant 2-forms over $M$, and $L M$ is the vector bundle of real $J$-skew-invariant 2-forms over $M$, respectively. Now, a linear connection $\nabla^{\prime}$ is called a Hermitian connection (or a Chern connection) on $M$ if both of the almost complex structure $J$ and the Riemannian metric are parallel with respect to $\nabla^{\prime}$. Let $W$ be a $(1,2)$-tensor field on $M$ satisfying the following condition:

$$
g(W(X, Y), Z)+g(W(X, Z), Y)=0
$$

for any $X, Y, Z \in \mathfrak{X}(M)$. Then, it is known that any Hermitian connection $\nabla^{\prime}$ is given by

$$
\nabla_{X}^{\prime} Y=\nabla_{X} Y+\frac{1}{2}\left(-J\left(\nabla_{X}\right) Y+W(X, Y)-J W(X, J Y)\right)
$$

for $X, Y \in \mathfrak{X}(M)$ [19]. We denote by $R^{\prime}$ the curvature tensor of the linear connection defined by (2.7) and by $\gamma_{1}^{\prime}$ the first Chern form corresponding to the linear connection $\nabla^{\prime}$. Then, we see that the first Chern form $\gamma_{1}^{\prime}=\left(\gamma_{i j}^{\prime}\right)$ is given by

$$
8 \pi \gamma_{i j}^{\prime}=R_{i j k}^{\prime}{ }^{l} J_{l}^{k}
$$

[9]. Then, from (2.6), (2.7) and (2.8), by direct calculation, we have the following equality:

$$
\gamma_{1}^{\prime}=\frac{1}{8 \pi}(-\phi+2 \psi-2 d \eta)
$$

where $\phi$ and $\psi$ are 2-forms on $M$ defined respectively by

$$
\phi(X, Y)=\operatorname{tr}\left(Z \rightarrow J\left(\nabla_{X} J\right)\left(\nabla_{Y} J\right) Z\right),
$$

$$
\psi(X, Y)=\operatorname{tr}(Z \rightarrow J R(X, Y) Z)
$$

for $X, Y, Z \in \mathfrak{X}(M)$ [9], and further $\eta=\left(\eta_{i}\right)$ is the 1-form on $M$ defined by

$$
\eta_{i}=-W_{i k}^{l} J_{l}^{k} \text {. }
$$

In the case where $M$ is compact, the first Chern class $c_{1}(M)$ of $M$ is given by the de Rham class $\left[\gamma_{1}^{\prime}\right] \in H^{2}(M ; \mathbb{R})$ due to the Chern-Weil theory. Thus, from the equality (2.9) with (2.12), we see that the first Chern class $c_{1}(M)$ of $M$ does not depend the choice of the (1,2)-tensor field $W$ with (2.6) on 
$M$. Therefore, it seems sufficiently enough to discuss based on the first Chern form $\gamma_{1}^{\prime}$ corresponding to $W=0$, namely the first Chern form $\gamma_{1}$ given by the following

$$
\gamma_{1}=\frac{1}{8 \pi}(-\phi+2 \psi)
$$

From (2.13), when $\operatorname{dim} M=4$, it follows that the first Chern number $c_{1}(M)^{2}$ of $M$ is given by

$$
c_{1}(M)^{2}=\left[\gamma_{1} \wedge \gamma_{1}\right] \in H^{4}(M ; \mathbb{R})(\simeq \mathbb{R}) .
$$

\section{Integral formula for $c_{1}(M)^{2}$}

Let $M=(M, g, J)$ be a compact almost Hermitian surface. Then, from (2.13) and (2.14), we see that the first Chern number $c_{1}(M)^{2}$ of $M$ is given by the following integral:

$$
c_{1}(M)^{2}=\int_{M} \gamma_{1} \wedge \gamma_{1}=\frac{1}{64 \pi^{2}} \int_{M}(\phi \wedge \phi-4 \phi \wedge \psi+4 \psi \wedge \psi) .
$$

Now, let $\left\{e_{i}\right\}=\left\{e_{1}, e_{2}=J e_{1}, e_{3}, e_{4}=J e_{3}\right\}$ be any local unitary basis and $\left\{e^{i}\right\}$ be local dual basis of $\left\{e_{i}\right\}$. Then, from (2.4) and (2.5), we see that the following equality

$$
\nabla \omega=\alpha \otimes \frac{1}{\sqrt{2}}\left(e^{1} \wedge e^{3}-e^{2} \wedge e^{4}\right)+\beta \otimes \frac{1}{\sqrt{2}}\left(e^{1} \wedge e^{4}+e^{2} \wedge e^{3}\right)
$$

holds for some local 1 -forms $\alpha$ and $\beta$. From (2.4) and (3.2), we get

$$
\begin{aligned}
\alpha_{i} & =-\sqrt{2} \nabla_{i} J_{13}=\sqrt{2} \nabla_{i} J_{24}, \\
\beta_{i} & =-\sqrt{2} \nabla_{i} J_{14}=-\sqrt{2} \nabla_{i} J_{23},
\end{aligned}
$$

where $\alpha_{i}=\alpha\left(e_{i}\right)$ and $\beta_{i}=\beta\left(e_{i}\right), i=1,2,3,4$. From (2.10) and (3.3), we get also

$$
\begin{array}{ll}
\phi_{12}=2\left(\alpha_{2} \beta_{1}-\alpha_{1} \beta_{2}\right), & \phi_{13}=2\left(\alpha_{3} \beta_{1}-\alpha_{1} \beta_{3}\right), \\
\phi_{14}=2\left(\alpha_{4} \beta_{1}-\alpha_{1} \beta_{4}\right), & \phi_{23}=2\left(\alpha_{3} \beta_{2}-\alpha_{2} \beta_{3}\right), \\
\phi_{24}=2\left(\alpha_{4} \beta_{2}-\alpha_{2} \beta_{4}\right), & \phi_{34}=2\left(\alpha_{4} \beta_{3}-\alpha_{3} \beta_{4}\right) .
\end{array}
$$

From (3.4), we have

$$
\phi=-2 \alpha \wedge \beta \text {. }
$$

Thus, from (3.5), we have immediately

$$
\phi \wedge \phi=0 .
$$

On the other hand, from (2.11), we have

$$
\psi(X, Y)=-2 \rho^{*}(X, J Y)
$$


for $X, Y \in \mathfrak{X}(M)$. Thus, from (3.7), we get

$$
\begin{aligned}
& \psi_{12}=2 \rho^{*}{ }_{11}, \quad \psi_{13}=-2 \rho^{*}{ }_{14}, \\
& \psi_{14}=2 \rho^{*}{ }_{13}, \quad \psi_{23}=-2 \rho^{*}{ }_{24}=-2 \rho^{*}{ }_{31}, \\
& \psi_{24}=2 \rho^{*}{ }_{23}=-2 \rho^{*}{ }_{41}, \quad \psi_{34}=2 \rho^{*}{ }_{33} .
\end{aligned}
$$

Thus, from (3.8), we have

$$
\begin{aligned}
\psi \wedge \psi & =2\left(\psi_{12} \psi_{34}-\psi_{13} \psi_{24}+\psi_{14} \psi_{23}\right) d v_{g} \\
& =8\left(\rho_{11}^{*} \rho_{33}^{*}+\rho_{14}^{*} \rho_{23}^{*}-\rho_{13}^{*} \rho_{24}^{*}\right) d v_{g} \\
& =\left\{\left(\tau^{*}\right)^{2}-2 \sum \rho_{i j}^{*} \rho_{j i}^{*}\right\} d v_{g} .
\end{aligned}
$$

Further, from (3.3), (3.4) and (3.8), we have

$$
\begin{aligned}
\phi \wedge \psi & =\left(\phi_{12} \psi_{34}-\phi_{13} \psi_{24}+\phi_{14} \psi_{23}+\phi_{34} \psi_{12}-\phi_{24} \psi_{13}+\phi_{23} \psi_{14}\right) d v_{g} \\
& =2\left\{\phi_{12} \rho_{33}^{*}-\phi_{13} \rho_{23}^{*}-\phi_{14} \rho_{24}^{*}+\phi_{34} \rho_{11}^{*}+\phi_{24} \rho_{14}^{*}+\phi_{23} \rho_{13}^{*}\right\} d v_{g} \\
& =\left\{\sum \rho_{i j}^{*}\left(\nabla_{j} J_{a b}\right)\left(\nabla_{s} J_{a t}\right) J_{i}{ }^{s} J_{b}{ }^{t}+\frac{\tau^{*}}{2} \sum\left(\nabla_{c} J_{a b}\right)\left(\nabla_{s} J_{t a}\right) J_{b}{ }^{t} J_{c}{ }^{s}\right\} d v_{g} .
\end{aligned}
$$

Therefore, from (3.6), (3.9) and (3.10), we have finally the following.

Theorem 1. Let $M=(M, g, J)$ be a compact almost Hermitian surface. Then the first Chern number $c_{1}(M)^{2}$ is given by the following integral formula:

$$
\begin{aligned}
c_{1}(M)^{2}=\frac{1}{16 \pi^{2}} \int_{M}\{ & \left(\tau^{*}\right)^{2}-2 \sum \rho_{i j}^{*} \rho_{j i}^{*}-\sum \rho_{i j}^{*}\left(\nabla_{j} J_{a b}\right)\left(\nabla_{s} J_{a t}\right) J_{i}{ }^{s} J_{b}{ }^{t} \\
& \left.-\frac{\tau^{*}}{2} \sum\left(\nabla_{c} J_{a b}\right)\left(\nabla_{s} J_{t a}\right) J_{b}{ }^{t} J_{c}{ }^{s}\right\} d v_{g} .
\end{aligned}
$$

Remark 1. In [16], Sekigawa also gave another integral formula for the first Chern number $c_{1}(M)^{2}$ of a compact almost Hermitian manifold $M=(M, g, J)$ by using the Lee-form and the Nijenhuis tensor of $J$ (for the definitions of the Lee-form and the Nijenhuis tensor, see $\S 5$ ).

\section{1-parameter deformations of $(g, J)$}

Let $M$ be a $2 n$-dimensional compact, orientable, smooth manifold admitting an almost complex structure. We denote by $\mathcal{M}(M)$ the space of all Riemannian metrics on $M$ and by $\Omega_{n d}^{2}(M)$ the space of all non-degenerate 2-forms on $M$. We also denote by $\mathcal{A H}(M)$ the space of all almost Hermitian structures on $M$. By our assumption, the space $\mathcal{A H}(M)$ is non-empty. It is well-known that the spaces $\mathcal{M}(M), \Omega_{n d}^{2}(M)$ and $\mathcal{A H}(M)$ are contractible Frechet manifolds. Further, we can check that $\mathcal{A H}(M)$ can be identified with a subspace of the product space $\mathcal{M}(M) \times \Omega_{n d}^{2}(M)$ by the map $\iota: \mathcal{A H}(M) \rightarrow \mathcal{M}(M) \times \Omega_{n d}^{2}(M)$ defined by $\iota:(g, J) \longmapsto(g, \omega)$, where $\omega$ is the Kähler form of $(g, J)$. Alternatively, in the sequel, we identify the space $\mathcal{A H}(M)$ with the space $\iota(\mathcal{A H}(M))$ under the map $\iota$. Now, let $(g, J)$ be any point of $\mathcal{A H}(M)$ and $\gamma(t)=(g(t), J(t))(|t|<\varepsilon, \varepsilon>0)$ 
be a smooth curve on $\mathcal{A H}(M)$ through $\gamma(0)=(g(0), J(0))=(g, J)$. We call the curve $\gamma(t)=(g(t), J(t))$ also a one-parameter deformation of $(g, J)$. We set $\alpha(t)=\omega(t)-\omega$, where $\omega(t)$ is the Kähler form of $(g(t), J(t))$. Let $\left(U ; x^{1}, x^{2}, \ldots, x^{2 n}\right)$ be a local coordinate system on a coordinate neighborhood $U$ in $M$ with respect to the natural frame field $\left\{\partial_{i} \equiv \frac{\partial}{\partial x^{i}}\right\}_{i=1,2, \ldots, 2 n}$ on $U$, we set $g(t)\left(\partial_{i}, \partial_{j}\right)=g(t)_{i j}, J(t) \partial_{i}=J(t)_{i}{ }^{j} \partial_{j}, \alpha(t)\left(\partial_{i}, \partial_{j}\right)=\alpha(t)_{i j}$, and further

$$
\left.\frac{d}{d t}\right|_{t=0} g(t)_{i j}=h_{i j},\left.\quad \frac{d}{d t}\right|_{t=0} J(t)_{j}{ }^{i}=K_{j}{ }^{i},\left.\quad \frac{d}{d t}\right|_{t=0} \alpha(t)_{i j}=A_{i j} .
$$

Then, we see that $h=\left(h_{i j}\right), A=\left(A_{i j}\right)$ and $K=\left(K_{j}{ }^{i}\right)$ are a symmetric $(0,2)$-tensor field, a 2 -form and a $(1,1)$-tensor field on $M$, respectively. We may regard the pair $(h, K)$ (resp. $(h, A))$ as the tangent vector of the curve $\gamma(t)=(g(t), J(t))$ (resp. $\tilde{\gamma}(t)=\iota(\gamma(t))=(g(t), \omega(t)))$ at the point $\gamma(0)=(g(0), J(0))=(g, J) \in \mathcal{A H}(M)$ (resp. at the point $\tilde{\gamma}(0)=\iota(\gamma(0))=$ $(g(0), \omega(0))=(g, \omega))$. We have also

$$
\left.\frac{d}{d t}\right|_{t=0} g(t)^{i j}=-h^{i j}
$$

We denote the volume form of the metric $g(t)$ by $d v_{g(t)}$. Then, we have

$$
\left.\frac{d}{d t}\right|_{t=0} d v_{g(t)}=\frac{1}{2}\left(g^{i j} h_{i j}\right) d v_{g}
$$

We denote the Riemannian connection, the curvature tensor, the Ricci tensor, the scalar curvature of $g(t)$ by $\nabla(t), R(t), \rho(t)$ and $\tau(t)$ and further the Ricci $*-$ tensor and the *-scalar curvature of $(g(t), J(t))$ by $\rho^{*}(t)$ and $\tau^{*}(t)$, respectively. Let $\Gamma(t)_{i j}{ }^{k}$ the Christoffel's symbol of $\nabla(t)$. Then, we have

$$
\left.\frac{d}{d t}\right|_{t=0} \Gamma(t)_{i j}^{k}=\frac{1}{2} g^{k a}\left(\nabla_{i} h_{a j}+\nabla_{j} h_{i a}-\nabla_{a} h_{i j}\right) .
$$

Thus, from (4.4), we have further

$$
\begin{aligned}
& \left.\frac{d}{d t}\right|_{t=0} R(t)_{i j k}{ }^{l} \\
= & \frac{1}{2}\left(-R_{i j k}{ }^{a} h_{a}{ }^{l}+R_{i j a}{ }^{l} h_{k}{ }^{a}+\nabla_{i} \nabla_{k} h_{j}{ }^{l}-\nabla_{j} \nabla_{k} h_{i}{ }^{l}-\nabla_{i} \nabla^{l} h_{j k}+\nabla_{j} \nabla^{l} h_{i k}\right),
\end{aligned}
$$

$$
\begin{aligned}
& \left.\quad \frac{d}{d t}\right|_{t=0} \rho(t)_{i j} \\
& =\frac{1}{2}\left(-R_{a i j}{ }^{b} h_{b}{ }^{a}+\rho_{i a} h_{j}{ }^{a}+\nabla_{a} \nabla_{j} h_{i}{ }^{a}-\nabla_{i} \nabla_{j} h_{a}{ }^{a}-\nabla^{a} \nabla_{a} h_{i j}+\nabla_{i} \nabla_{a} h_{j}{ }^{a}\right), \\
& \left.\frac{d}{d t}\right|_{t=0} \tau(t)=-\rho_{i j} h^{i j}+\nabla^{i} \nabla^{j} h_{i j}-\nabla^{i} \nabla_{i} h_{a}{ }^{a} .
\end{aligned}
$$


Here, since $\gamma(t)=(g(t), J(t)) \in \mathcal{A H}(M)$, from (4.1), we have the following equalities [13]:

$$
\begin{gathered}
K_{a}{ }^{i}{J_{j}}^{a}+J_{a}{ }^{i} K_{j}{ }^{a}=0, \\
h_{i j}=h_{a b} J_{i}{ }^{a} J_{j}{ }^{b}+K_{i a} J_{j}{ }^{a}+J_{i a} K_{j}{ }^{a}, \\
K_{j}{ }^{i}=-h_{a}{ }^{i} J_{j}{ }^{a}-A_{j}{ }^{i} .
\end{gathered}
$$

From (4.9) and (4.10), we have

$$
h_{i j}=-h_{a b} J_{i}{ }^{a} J_{j}^{b}+J_{i}^{a} A_{a j}+J_{j}{ }^{a} A_{a i} .
$$

From (4.10) and (4.11), we have also

$$
K_{j}{ }^{i}=h_{j}{ }^{a} J_{a}{ }^{i}-A_{b}{ }^{a} J_{a}{ }^{i} J_{j}{ }^{b} .
$$

Conversely, for any pair $(h, A)$ satisfying (4.11), we define $(1,1)$-tensor field $K=\left(K_{j}{ }^{i}\right)$ by (4.12). Then, we can check that the pair $(h, K)$ satisfies $(4.8)$ $\sim(4.10)$, and that the pair corresponds to the pair $(h, K)$ under the map $\iota$ coincides with the pair $(h, A)$. Further, Gil-Medrano and Michor [8] showed that, for any $(g, \omega) \in \iota(\mathcal{A H}(M))$, a pair $(h, A)$ of a symmetric $(0,2)$-tensor $h$ and a 2 -form $A$ on $M$ is a tangent vector of the space $\iota(\mathcal{A H}(M))$ at $(g, \omega)$ if and only if $(h, A)$ satisfies the equality (4.11). Now, from $(2.2),(4.2),(4.4)$ and (4.5), taking account of (4.10), (4.11) and (4.12), we have also

$$
\begin{aligned}
\left.\frac{d}{d t}\right|_{t=0} \rho^{*}(t)_{i j}= & \rho_{i a}^{*} h_{j}{ }^{a}-\frac{1}{2} R_{i u a}{ }^{b} J_{j}{ }^{u} J^{a c} h_{b c}-\frac{1}{2} J^{a b} J_{j}{ }^{c} \nabla_{i} \nabla_{a} h_{b c} \\
& +\frac{1}{2} J^{a b} J_{j}^{c} \nabla_{c} \nabla_{a} h_{b i}+\frac{1}{2}\left(2 J_{j}{ }^{q} \rho^{*}{ }_{i}{ }^{p}-J_{j}{ }^{u} J^{p a} J^{q b} R_{i u a b}\right) A_{p q}
\end{aligned}
$$

$$
\begin{aligned}
\left.\frac{d}{d t}\right|_{t=0} \tau^{*}(t) & =\rho_{a b}^{*} h^{a b}-J^{i a} J^{j b} \nabla_{a} \nabla_{b} h_{i j}-2 J^{i p} \rho_{i q}^{*} A_{p}{ }^{q}, \\
\left.\frac{d}{d t}\right|_{t=0} \nabla(t)_{i} J(t)_{j}{ }^{k}= & -h_{a}{ }^{k} \nabla_{i} J_{j}{ }^{a}+\frac{1}{2} J_{j}{ }^{a}\left(\nabla_{a} h_{i}{ }^{k}-\nabla_{i} h_{a}{ }^{k}-\nabla^{k} h_{i a}\right) \\
& -\frac{1}{2} J_{a}{ }^{k}\left(\nabla_{i} h_{j}{ }^{a}+\nabla_{j} h_{i}{ }^{a}-\nabla^{a} h_{i j}\right)-\nabla_{i} A_{j}{ }^{k} .
\end{aligned}
$$

In [8], Gil-Medrano and Michor also proved that the tangent space

$$
T_{(g, \omega)} \iota(\mathcal{A H}(M))
$$

of the space $\iota(\mathcal{A H}(M))$ at any point $(g, \omega)$ can be decomposed as follows:

$$
T_{(g, \omega)} \iota(\mathcal{A H}(M))=\mathcal{N}_{(g, \omega)}^{1} \oplus \mathcal{N}_{(g, \omega)}^{2} \oplus \mathcal{N}_{(g, \omega)}^{3},
$$


where $\mathcal{N}_{(g, \omega)}^{1}, \mathcal{N}_{(g, \omega)}^{2}$ and $\mathcal{N}_{(g, \omega)}^{3}$ are the subspaces of $T_{(g, \omega)}\left(\mathcal{M}(M) \times \Omega_{n d}^{2}(M)\right)$ defined by

$$
\begin{aligned}
& \mathcal{N}_{(g, \omega)}^{1}=\left\{(h, 0) \mid h_{\overline{i j}}=-h_{i j}\right\}, \\
& \mathcal{N}_{(g, \omega)}^{2}=\left\{(0, A) \mid A_{\bar{i} j}=A_{i \bar{j}}\right\}, \\
& \mathcal{N}_{(g, \omega)}^{3}=\left\{(h, A) \mid A_{i j}=-h_{\bar{i} j}, \quad h_{\bar{i} \bar{j}}=h_{i j}\right\} .
\end{aligned}
$$

Then, from $(4.8) \sim(4.12)$, we see that $(h, A) \in \mathcal{N}_{(g, \omega)}^{2} \cup \mathcal{N}_{(g, \omega)}^{3}$ if and only if $h=\left(h_{i j}\right)$ is expressed as follows:

$$
h_{i j}=\frac{1}{2}\left(A_{\overline{i j}}+A_{\overline{j i}}\right)
$$

for any 2 -form $A=\left(A_{i j}\right)$ on $M$. Let $(g, J)$ be any point of $\mathcal{A H}(M)$. Then, we call the deformation defined by a curve $\gamma(t)=(g(t), J(t))(|t|<\varepsilon, \varepsilon>0)$ in $\mathcal{A H}(M)$ through the point $(g(0), J(0))=(g, J)$ a Type $(m)$-deformation if the tangent vector $\frac{d \tilde{\gamma}}{d t}=\frac{d(\llcorner\circ \gamma)}{d t}$ belongs to the subspace $\mathcal{N}_{(g, \omega)}^{m}(m=1,2,3)$. In [13], we call the deformation defined by the curve $\gamma(t)=(g(t), J(t)) \in \mathcal{A H}(M)$ through the point $(g(0), J(0))=(g, J)$ the Blair-Ianus deformation of $(g, J)$ if the tangent vector $\frac{d \tilde{\gamma}}{d t}$ of the curve $\tilde{\gamma}=\iota \circ \gamma$ belongs to $\mathcal{N}_{(g(t), \omega(t))}^{1}$ for any $t(|t|<$ $\varepsilon)$, and also the Type (2)-deformation, Type (3)-deformation the deformation of Type (ii) in [13]. Gil-Medrano and Michor [8] provided explicit examples of a Blair-Ianus deformation, and also of two deformations of Type $(i i)$ in [13], one is of Type (2) and the other is of Type (3).

\section{Curvature identities}

Let $M=(M, g, J)$ be an almost Hermitian surface. Then, we see that there exists a 1 -form $\theta=\left(\theta_{i}\right)$ on $M$ satisfying the following equation [14]:

$$
d \omega=\theta \wedge \omega .
$$

The 1 -form $\theta$ is called the Lee-form of the almost Hermitian structure $(g, J)$ on $M$, which is locally expressed as

$$
\theta_{i}=-J_{i}^{j} \nabla_{a} J_{j}{ }^{a} .
$$

From (5.1), we have immediately

$$
d \theta \wedge \omega=0 .
$$

We may easily check that the equation (5.3) implies

$$
J^{i j} \nabla_{i} \theta_{j}=0 .
$$

By making use of (2.4)and (5.1), we have the following equality:

$$
2 \nabla_{i} J_{j k}=-\theta_{j} J_{i k}+\theta_{k} J_{i j}+J_{j}{ }^{a} \theta_{a} g_{i k}-J_{k}{ }^{a} \theta_{a} g_{i j}+N_{j k a} J_{i}^{a},
$$

[16], where $N=\left(N_{i j}{ }^{k}\right)$ is the Nijenhuis tensor of the almost complex structure $J$ defined by

$$
N(X, Y)=[J X, J Y]-[X, Y]-J[J X, Y]-J[X, J Y]
$$


for $X, Y \in \mathfrak{X}(M)$. First of all, we can show that the following curvature equality holds on any almost Hermitian surface $M$ :

$$
\rho^{*}{ }_{i j}+\rho^{*}{ }_{j i}-\rho_{i j}-\rho_{\overline{i j}}=\frac{1}{2}\left(\tau^{*}-\tau\right) g_{i j},
$$

[10]. Concerning Question A, the following question will naturally arise:

Question B. Can we derive curvature identities besides (5.7) from the integral formula in Theorem 1 for the Chern number of compact almost Hermitian surfaces?

We here recall the following Wu's Theorem:

Theorem 2 ([18]). Let $M=(M, g, J)$ be a compact almost Hermitian surface. Then the following equality holds:

$$
c_{1}(M)^{2}=2 \chi(M)+p_{1}(M),
$$

where $\chi(M)$ and $p_{1}(M)$ are the Euler number and the first Pontrjagin number of $M$, respectively.

From Theorem 2, we see that the first Chern number $c_{1}(M)^{2}$ of $M$ given by (3.11) in Theorem 1 is a topological invariant of $M$ by the Novikov's Theorem [15], and hence, $c_{1}(M)^{2}$ does not depend on the choice of almost Hermitian structures on $M$. We also notes that the second Chern number $c_{2}(M)$ of $M$ coincides with the Euler number $\chi(M)$ of $M$. Now, we set

$$
\begin{aligned}
\mathcal{F}(g, J)=\int_{M}\{ & \left(\tau^{*}\right)^{2}-2 \rho^{* i j} \rho_{j i}^{*}-g^{j l} g^{i k} \rho_{u k}^{*}\left(\nabla_{i} J_{a}{ }^{b}\right)\left(\nabla_{j} J_{s}{ }^{a}\right) J_{l}{ }^{u} J_{b}{ }^{s} \\
& \left.-\frac{1}{2} \tau^{*} g^{c l}\left(\nabla_{l} J_{a}{ }^{b}\right)\left(\nabla_{s} J_{u}{ }^{a}\right) J_{b}{ }^{u} J_{c}{ }^{s}\right\} d v_{g} .
\end{aligned}
$$

Then, from Theorem 1, we have

$$
c_{1}(M)^{2}=\frac{1}{16 \pi^{2}} \mathcal{F}(g, J),
$$

and $\mathcal{F}(g, J)$ is constant on the space $\mathcal{A H}(M)$. Let $(g, J)$ be any point of $\mathcal{A H}(M)$ and $\gamma(t)=(g(t), J(t))(|t|<\varepsilon)$ be any smooth curve in $\mathcal{A H}(M)$ through the point $(g(0), J(0))=(g, J)$. Thus, from Theorem 1 and Theorem 2, taking account of (5.8) and (5.10), we have

$$
\left.\frac{d}{d t}\right|_{t=0} \mathcal{F}(g(t), J(t))=0 .
$$

Here, from (4.3) and (4.14), we get

$$
\begin{aligned}
\left.\frac{d}{d t}\right|_{t=0} \int_{M}\left(\tau^{*}(t)\right)^{2} d v_{g(t)}=\int_{M} & {\left[\left\{2 \tau^{*} \rho^{* i j}-2 \nabla_{b} \nabla_{a}\left(\tau^{*} J^{i a} J^{j b}\right)+\frac{1}{2}\left(\tau^{*}\right)^{2} g^{i j}\right\} h_{i j}\right.} \\
& \left.-4 \tau^{*} J^{a p} \rho_{a}^{* q} A_{p q}\right] d v_{g} .
\end{aligned}
$$


From (4.2), (4.3) and (4.13), taking account of (2.3), we get

$$
\begin{aligned}
& \left.\frac{d}{d t}\right|_{t=0} \int_{M} \rho^{*}(t)^{i j} \rho^{*}(t)_{j i} d v_{g(t)} \\
= & \int_{M}\left[\left\{-\rho^{*}{ }_{l k} R_{a b}^{k}{ }^{i} J^{l a} J^{b j}-2 \nabla_{a} \nabla^{l}\left(\rho^{*}{ }_{k l} J^{a i} J^{k j}\right)+\frac{1}{2} \rho^{* a b} \rho_{b a}^{*} g^{i j}\right\} h_{i j}\right. \\
& \left.+\left(2 J^{l q} \rho^{*}{ }_{l k} \rho^{* k p}-\rho^{*}{ }_{l k} J^{l c} J^{p a} J^{q b} R_{c a b}^{k}\right) A_{p q}\right] d v_{g} .
\end{aligned}
$$

Further, from (4.2), (4.3), (4.13) and (4.15), taking account of (2.3) and the Green's Theorem, we get

$$
\begin{aligned}
& \left.\frac{d}{d t}\right|_{t=0} \int_{M} g(t)^{j l} J(t)_{l}{ }^{t} g(t)^{i k} \rho^{*}(t)_{t k}\left(\nabla(t)_{i} J(t)_{a}{ }^{b}\right)\left(\nabla(t)_{j} J(t)_{s}{ }^{a}\right) J(t)_{b}{ }^{s} d v_{g(t)} \\
= & \int_{M}\left[\left\{-J^{i v} \rho_{v}^{*}{ }_{v}^{w}\left(\nabla_{w} J_{a}{ }^{b}\right)\left(\nabla^{j} J_{u}{ }^{a}\right) J_{b}{ }^{u}-J^{w i} \rho^{* j c}\left(\nabla_{c} J_{a}{ }^{b}\right)\left(\nabla_{w} J_{u}{ }^{a}\right) J_{b}{ }^{u}\right.\right. \\
& -\frac{1}{2} J^{c v} R_{v w s}{ }^{i} J^{k w} J^{s j}\left(\nabla_{k} J_{a}{ }^{b}\right)\left(\nabla_{c} J_{u}{ }^{a}\right) J_{b}{ }^{u} \\
& +\nabla_{s} \nabla_{c}\left(J^{v j} J^{s i} J_{k}{ }^{c}\left(\nabla^{k} J_{a}{ }^{b}\right)\left(\nabla_{v} J_{u}{ }^{a}\right) J_{b}{ }^{u}\right) \\
& +3 J^{c v} \rho^{*}{ }_{v}^{k}\left(\nabla_{k} J_{a}{ }^{i}\right)\left(\nabla_{c} J^{j w}\right) J_{w}{ }^{a}+\frac{1}{2} J^{c v} \rho^{*}{ }_{v}{ }^{k}\left(\nabla_{k} J_{a}{ }^{b}\right)\left(\nabla_{c} J_{u}{ }^{a}\right) J_{b}{ }^{u} g^{i j} \\
& \left.-4 \nabla_{u}\left(J^{c v} \rho^{*}{ }_{v}^{i}\left(\nabla_{c} J^{j u}\right)\right)\right\} h_{i j}+\left\{J^{j v} J^{i q} \rho_{v}^{*}{ }_{v}^{p}\left(\nabla_{i} J_{a}{ }^{b}\right)\left(\nabla_{j} J_{u}{ }^{a}\right) J_{b}{ }^{u}\right. \\
& -\frac{1}{2} J^{j v} J^{i w} J^{p s} J^{q c} R_{v w s c}\left(\nabla_{i} J_{a}{ }^{b}\right)\left(\nabla_{j} J_{u}{ }^{a}\right) J_{b}{ }^{u}+2 \nabla_{i}\left(J^{j v} \rho^{*}{ }_{v}{ }^{i}\left(\nabla_{j} J_{w}{ }^{p}\right) J^{q w}\right) \\
& \left.\left.-\rho^{* q i}\left(\nabla_{i} J_{a}{ }^{b}\right)\left(\nabla^{p} J_{u}{ }^{a}\right) J_{b}{ }^{u}-J^{j v} \rho^{*}{ }_{v}{ }^{i}\left(\nabla_{i} J_{a}{ }^{p}\right)\left(\nabla_{j} J^{q a}\right)\right\} A_{p q}\right] d v_{g} .
\end{aligned}
$$

Similarly, from (4.2), (4.3), (4.14) and (4.15), we get

$$
\begin{aligned}
& \left.\frac{d}{d t}\right|_{t=0} \int_{M} \tau^{*}(t) g(t)^{c u}\left(\nabla(t)_{u} J(t)_{a}{ }^{b}\right)\left(\nabla(t)_{s} J(t)_{v}{ }^{a}\right) J(t)_{b}{ }^{v} J(t)_{c}{ }^{s} d v_{g(t)} \\
= & \int_{M}\left[\left\{\rho^{* i j}\left(\nabla_{c} J_{a}{ }^{b}\right)\left(\nabla_{u} J_{v}{ }^{a}\right) J_{b}{ }^{v} J^{c u}-\nabla_{l} \nabla_{k}\left(J^{i k} J^{j l}\left(\nabla_{c} J_{a}{ }^{b}\right)\left(\nabla_{u} J_{v}{ }^{a}\right) J_{b}{ }^{v} J^{c u}\right)\right.\right. \\
& -4 \nabla_{c}\left(\tau^{*}\left(\nabla_{u} J^{j c}\right) J^{i u}\right)-3 \tau^{*}\left(\nabla_{c} J^{j b}\right)\left(\nabla_{u} J_{v}{ }^{i}\right) J_{b}{ }^{v} J^{c u} \\
& \left.-2 \tau^{*}\left(\nabla^{j} J_{a}{ }^{b}\right)\left(\nabla_{u} J_{v}{ }^{a}\right) J_{b}{ }^{v} J^{i u}+\frac{1}{2} \tau^{*}\left(\nabla_{c} J_{a}{ }^{b}\right)\left(\nabla_{u} J_{v}{ }^{a}\right) J_{b}{ }^{v} J^{c u} g^{i j}\right\} h_{i j} \\
& +\left\{-2 J^{i p} \rho^{*}{ }_{i}^{q}\left(\nabla_{c} J_{a}{ }^{b}\right)\left(\nabla_{u} J_{v}{ }^{a}\right) J_{b}{ }^{v} J^{c u}-\tau^{*}\left(\nabla_{c} J_{a}{ }^{p}\right)\left(\nabla_{u} J^{q a}\right) J^{c u}\right. \\
& \left.\left.-\tau^{*}\left(\nabla^{p} J_{a}{ }^{b}\right)\left(\nabla^{q} J_{v}{ }^{a}\right) J_{b}{ }^{v}+2 \nabla_{u}\left(\tau^{*}\left(\nabla_{c} J^{q b}\right) J_{b}{ }^{p} J^{c u}\right)\right\} A_{p q}\right] d v_{g} .
\end{aligned}
$$

Thus, from $(5.11),(5.12),(5.13),(5.14)$ and (5.15), we have

$$
\int_{M}\left\{T^{\prime i j} h_{i j}+S^{\prime p q} A_{p q}\right\} d v_{g}=0
$$




$$
\begin{aligned}
T^{\prime i j}= & J^{i v} \rho_{v}^{* w}\left(\nabla_{w} J_{a}{ }^{b}\right)\left(\nabla^{j} J_{u}{ }^{a}\right) J_{b}{ }^{u}+J^{w i} \rho^{* j c}\left(\nabla_{c} J_{a}{ }^{b}\right)\left(\nabla_{w} J_{u}{ }^{a}\right) J_{b}{ }^{u} \\
& +4 \nabla_{u}\left(J^{c v} \rho_{v}^{* i}\left(\nabla_{c} J^{j u}\right)\right)+\frac{1}{2} J^{c v} R_{v w s}{ }^{i} J^{d w} J^{s j}\left(\nabla_{d} J_{a}{ }^{b}\right)\left(\nabla_{c} J_{u}{ }^{a}\right) J_{b}{ }^{u} \\
& -\nabla_{s} \nabla_{c}\left(J^{d j} J^{s i} J_{k}{ }^{c}\left(\nabla^{k} J_{a}{ }^{b}\right)\left(\nabla_{d} J_{u}{ }^{a}\right) J_{b}{ }^{u}\right) \\
& -3 J^{c v} \rho_{v}^{* d}\left(\nabla_{d} J_{a}{ }^{i}\right)\left(\nabla_{c} J^{j w}\right) J_{w}{ }^{a} \\
& -\frac{1}{2} J^{c v} \rho_{v}^{* d}\left(\nabla_{d} J_{a}{ }^{b}\right)\left(\nabla_{c} J_{u}{ }^{a}\right) J_{b}{ }^{u} g^{i j}-\frac{1}{2} \rho^{* i j}\left(\nabla_{c} J_{a}{ }^{b}\right)\left(\nabla_{u} J_{v}{ }^{a}\right) J_{b}{ }^{v} J^{c u} \\
& +\frac{1}{2} \nabla_{l} \nabla_{k}\left(J^{i k} J^{j l}\left(\nabla_{c} J_{a}{ }^{b}\right)\left(\nabla_{u} J_{v}{ }^{a}\right) J_{b}{ }^{v} J^{c u}\right)+2 \nabla_{c}\left(\tau^{*}\left(\nabla_{u} J^{j c}\right) J^{i u}\right) \\
& +\frac{3}{2} \tau^{*}\left(\nabla_{c} J^{j b}\right)\left(\nabla_{u} J_{v}{ }^{i}\right) J_{b}{ }^{v} J^{c u}+\tau^{*}\left(\nabla^{j} J_{a}{ }^{b}\right)\left(\nabla_{u} J_{v}{ }^{a}\right) J_{b}{ }^{v} J^{i u} \\
& -\frac{1}{4} \tau^{*}\left(\nabla_{c} J_{a}{ }^{b}\right)\left(\nabla_{u} J_{v}{ }^{a}\right) J_{b}{ }^{v} J^{c u} g^{i j}+2 \rho_{l k}^{*} R^{k}{ }_{a b}{ }^{i} J^{l a} J^{b j} \\
& +4 \nabla_{a} \nabla^{l}\left(\rho_{k l}^{*} J^{a i} J^{k j}\right)-\rho^{* a b} \rho_{b a}^{*} g^{i j}+2 \tau^{*} \rho^{* i j} \\
& -2 \nabla_{b} \nabla_{a}\left(\tau^{*} J^{i a} J^{j b}\right)+\frac{1}{2}\left(\tau^{*}\right)^{2} g^{i j},
\end{aligned}
$$

and

$$
\begin{aligned}
S^{\prime p q}= & -J^{j v} J^{i q} \rho_{v}^{* p}\left(\nabla_{i} J_{a}{ }^{b}\right)\left(\nabla_{j} J_{u}{ }^{a}\right) J_{b}{ }^{u} \\
& +\frac{1}{2} J^{j v} J^{i w} J^{p c} J^{q d} R_{v w c d}\left(\nabla_{i} J_{a}{ }^{b}\right)\left(\nabla_{j} J_{u}{ }^{a}\right) J_{b}{ }^{u} \\
& -2 \nabla_{i}\left(J^{j v} \rho_{v}^{* i}\left(\nabla_{j} J_{w}{ }^{p}\right) J^{q w}\right)+\rho^{* q i}\left(\nabla_{i} J_{a}{ }^{b}\right)\left(\nabla^{p} J_{u}{ }^{a}\right) J_{b}{ }^{u} \\
& +J^{j v} \rho_{v}^{* i}\left(\nabla_{i} J_{a}{ }^{p}\right)\left(\nabla_{j} J^{q a}\right)+J^{i p} \rho_{i}^{* q}\left(\nabla_{c} J_{a}{ }^{b}\right)\left(\nabla_{u} J_{v}{ }^{a}\right) J_{b}{ }^{v} J^{c u} \\
& +\frac{1}{2} \tau^{*}\left(\nabla_{c} J_{a}{ }^{p}\right)\left(\nabla_{u} J^{q a}\right) J^{c u}+\frac{1}{2} \tau^{*}\left(\nabla^{p} J_{a}{ }^{b}\right)\left(\nabla^{q} J_{v}{ }^{a}\right) J_{b}{ }^{v} \\
& -\nabla_{u}\left(\tau^{*}\left(\nabla_{c} J^{q b}\right) J_{b}{ }^{p} J^{c u}\right)-4 J^{l q} \rho_{l k}^{*} \rho^{* k p} \\
& +2 \rho_{l k}^{*} J^{l c} J^{p a} J^{q b} R^{k}{ }_{c a b}-4 \tau^{*} J^{a p} \rho_{a}^{* q} .
\end{aligned}
$$

We define $T=\left(T_{i j}\right)$ and $S=\left(S_{i j}\right)$ by

$$
T_{i j}=g_{i a} g_{j b} T^{a b}, \quad \text { where } \quad T^{i j}=\frac{1}{2}\left(T^{\prime i j}+T^{\prime j i}\right),
$$

and

$$
S_{i j}=g_{i p} g_{j q} S^{p q}, \quad \text { where } \quad S^{p q}=\frac{1}{2}\left(S^{\prime p q}-S^{\prime q p}\right),
$$

respectively. Then, the integral equation (5.16) is rewritten as

$$
\int_{M}\left\{T^{i j} h_{i j}+S^{p q} A_{p q}\right\} d v_{g}=0 .
$$


Lemma $3([2])$. Let $B=\left(B^{i j}\right)$ be a symmetric $(0,2)$-tensor field on $M$. Then

$$
\int_{M} \sum_{i, j} B^{i j} D_{i j} d v_{g}=0
$$

for all symmetric $J$-skew-invariant $(0,2)$-tensor field $D$ if and only if $B=\left(B_{i j}\right)$ $=\left(g_{i a} g_{j b} B^{a b}\right)$ is J-invariant.

Now, let $M=(M, g, J)$ be any compact almost Hermitian surface and consider any Type (1)-deformation of $(g, J)$. Then, from (5.21), taking account of Lemma 3 , we see that the tensor field $T$ is J-invariant. Similarly, considering any Type (2) or Type (3)-deformation of $(g, J)$, from (4.17) and (5.21), we have

$$
\begin{aligned}
& \int_{M}\left(\frac{1}{2} T^{i j} J_{i}{ }^{a} A_{a j}+\frac{1}{2} T^{i j} J_{j}{ }^{a} A_{a i}+S^{i j} A_{i j}\right) d v_{g} \\
= & \int_{M}\left(\frac{1}{2} T^{a j} J_{a}{ }^{i}-\frac{1}{2} T^{i a} J_{a}^{j}+S^{i j}\right) A_{i j} d v_{g} \\
= & \int_{M}\left(-T^{i a} J_{a}{ }^{j}+S^{i j}\right) A_{i j} d v_{g}=0
\end{aligned}
$$

for any 2 -form $A=\left(A_{i j}\right)$ on $M$. Thus, from (5.22), we have

$$
T_{i \bar{j}}+S_{i j}=0 \text {. }
$$

Therefore, summing up the above arguments, we have the following:

Theorem 4. Let $M=(M, g, J)$ be a compact almost Hermitian surface and $T=\left(T_{i j}\right), S=\left(S_{i j}\right)$ be the symmetric $(0,2)$-tensor field and the skew-symmetric $(0,2)$-tensor field defined by (5.19) with (5.17) and (5.20) with (5.18), respectively. Then, $T_{i j}=T_{\bar{i} \bar{j}}, T_{i \bar{j}}+S_{i j}=0$ and hence, $S_{i j}=S_{\bar{i} \bar{j}}$ hold on any compact almost Hermitian surface $M$.

Remark 2. Taking use of the equalities (5.4) and (5.5), it seems principally possible to rewrite the identities obtained in Theorem 1 in terms of the curvature tensor $R$, the almost complex structure $J$, the Lee-form $\theta$ and their covariant derivatives. It seems worthwhile to write down their explicit forms in view of their applications. In the present paper, we shall discuss this for a Kähler surface in the following section.

\section{An application}

In this section, we provide an application of Theorem 4 . Let $M=(M, J, g)$ be a Kähler surface. Then, it is well-known that $M$ satisfies the following identity:

$$
R_{i j k l}=R_{i j a b} J_{k}^{a} J_{l}^{b} .
$$

From (6.1), we see easily that the curvature identity (5.7) is trivial. Thus, it is natural to ask the following related to the Question B:

Question C. Do there exist curvature identities besides (6.1)? 
Concerning Question C, we shall prove the following theorem.

Theorem 5. Let $M=(M, g, J)$ be a compact Kähler surface. Then, in addition to the identity (6.1), the identity

$$
2 \rho_{i}^{a} \rho_{j a}-\tau \rho_{i j}-\frac{1}{2}\left(|\rho|^{2}-\frac{\tau^{2}}{2}\right) g_{i j}=0
$$

holds on $M$.

Proof. Since $M$ is Kähler, from (5.17), taking account of (6.1), we obtain

$$
\begin{aligned}
T_{i \bar{j}}^{\prime}= & 2 \rho^{l k} R_{k \bar{l} j i}-2 J_{i}{ }^{a} \nabla_{a} \nabla_{j} \tau+2 J_{i}{ }^{a} \nabla_{j} \nabla_{a} \tau \\
& -|\rho|^{2} J_{j i}+2 \tau \rho_{i \bar{j}}+\frac{1}{2} \tau^{2} J_{j i} \\
= & -2 \rho^{k l} R_{k \bar{l} i j}+|\rho|^{2} J_{i j}+2 \tau \rho_{i \bar{j}}-\frac{\tau^{2}}{2} J_{i j} .
\end{aligned}
$$

Similarly, from (5.18), we get

$$
\begin{aligned}
S_{i j}^{\prime} & =-4 J_{l j} \rho^{l k} \rho_{k i}+2 \rho^{l k} R_{k \bar{l} i j}+4 \tau \rho_{\bar{i} j} \\
& =4 \rho_{\bar{j}}^{a} \rho_{a i}+2 \rho^{k l} R_{k \bar{l} i j}+4 \tau \rho_{\bar{i} j} .
\end{aligned}
$$

From (6.4), we see that $S^{\prime}=\left(S_{i j}^{\prime}\right)$ is skew-symmetric and $J$-invariant and hence, we have

$$
\begin{aligned}
S_{i j} & =S_{i j}^{\prime} \\
& =4 \rho_{\bar{j}}^{a} \rho_{i a}+2 \rho^{k l} R_{k \bar{l} i j}+4 \tau \rho_{\bar{i} j} .
\end{aligned}
$$

On the other hand, from (6.3), we have

$$
T_{i j}^{\prime}=2 \rho^{k l} R_{k \bar{l} i \bar{j}}-|\rho|^{2} g_{i j}+2 \tau \rho_{i j}+\frac{\tau^{2}}{2} g_{i j} .
$$

Then, from (6.6), we see that $T^{\prime}=\left(T_{i j}^{\prime}\right)$ is symmetric and $J$-invariant, and hence we have

$$
\begin{aligned}
T_{i j} & =T_{i j}^{\prime} \\
& =2 \rho^{k l} R_{k \bar{l} i \bar{j}}-|\rho|^{2} g_{i j}+2 \tau \rho_{i j}+\frac{\tau^{2}}{2} g_{i j} .
\end{aligned}
$$

Thus, from Theorem 4, (6.5) and (6.7), we have

$$
\begin{aligned}
0 & =T_{i \bar{j}}+S_{i j} \\
& =|\rho|^{2} J_{i j}-\frac{\tau^{2}}{2} J_{i j}-2 \tau \rho_{i \bar{j}}+4 \rho_{i a} \rho_{\bar{j}}^{a} .
\end{aligned}
$$

There, from (6.8), we have finally

and hence

$$
|\rho|^{2} g_{i j}-\frac{\tau^{2}}{2} g_{i j}+2 \tau \rho_{i j}-4 \rho_{i a} \rho_{j}{ }^{a}=0,
$$

$$
2 \rho_{i a} \rho_{j}{ }^{a}-\tau \rho_{i j}-\frac{1}{2}|\rho|^{2} g_{i j}+\frac{\tau^{2}}{4} g_{i j}=0 .
$$


Thus, completes the proof of Theorem 5 .

We here recall the following curvature identity on any 4-dimensional Riemannian manifold $(M, g)[3]$ :

$$
R_{a b c i} R_{j}^{a b c}-2 \rho_{i}{ }^{a} \rho_{j a}-2 \rho^{a b} R_{i a b j}+\tau \rho_{i j}-\left(\frac{1}{4}|R|^{2}-|\rho|^{2}+\frac{\tau^{2}}{4}\right) g_{i j}=0 .
$$

Thus, from Theorem 5 and (6.10), we have the following.

Corollary 6. Let $M=(M, g, J)$ be a compact Kähler surface. Then, in addition to the identity (6.1), the identity

$$
R_{a b c i} R_{j}^{a b c}-2 \rho^{a b} R_{i a b j}-\frac{1}{4}\left(|R|^{2}-2|\rho|^{2}\right) g_{i j}=0
$$

holds on $M$.

As we discussed in the introduction, the curvature identity that holds on any compact Riemannian manifold can be extended to any Riemannian manifold. Similarly we wonder if a curvature identity (derived from the first Chern number) that holds on any compact almost Hermitian manifold can be extended to any almost Hermitian manifold. Thus we raise the following question:

Question D. Do the identities in Theorem 4 hold without compactness assumption?

\section{References}

[1] M. Berger, Quelques formules de variation pour une structure riemannienne, Ann. Sci. École Norm. Sup. (4) 3 (1970), 285-294.

[2] D. E. Blair and S. Ianus, Critical associated metrics on symplectic manifolds, Nonlinear problems in geometry (Mobile, Ala., 1985), 23-29, Contemp. Math., 51, Amer. Math. Soc., Providence, RI, 1986.

[3] Y. Euh, J. H. Park, and K. Sekigawa, A Curvature identity on a 4-dimensional Riemannian manifold, Results in Mathematics, DOI: 10.1007/s00025-011-0164-3.

[4] _ A generalization of 4-dimensional Einstein manifold, to appear in Mathematica Slovaca.

[5] _ Critical metrics for quadratic functionals in the curvature on 4-dimensional manifolds, Differential Geom. Appl. 29 (2011), no. 5, 642-646.

[6] P. Gilkey, J. H. Park, and K. Sekigawa, Universal curvature identities, Differential Geom. Appl. 29 (2011), no. 6, 770-778.

7] _ Universal curvature identities II, J. Geom. Phys. 62 (2012), no. 4, 814-825.

[8] O. Gil-Medrano and P. W. Michor, Geodesics on spaces of almost Hermitian structures, Israel J. Math. 88 (1994), no. 1-3, 319-332.

[9] A. Gray, M. Barros, A. M. Naveira, and L. Vanhecke, The Chern numbers of holomorphic vector bundles and formally holomorphic connections of complex vector bundles over almost complex manifolds, J. Reine Angew. Math. 314 (1980), 84-98.

[10] A. Gray and L. M. Hervella, The sixteen classes of almost Hermitian manifolds, Ann. Mat. Pura Appl. (4) 123 (1980), 35-58.

[11] G. M. Kuz'mina, Some generalizations of the Riemann spaces of Einstein, Math. Notes 16 (1974), 961-963; translation from Mat. Zametki 16 (1974), 619-622.

[12] M. L. Labbi, Variational properties of the Gauss-Bonnet curvatures, Calc. Var. Partial Differential Equations 32 (2008), no. 2, 175-189. 
[13] J. C. Lee, J. H. Park, and K. Sekigawa, Notes on critical almost Hermitian structures, Bull. Korean Math. Soc. 47 (2010), no. 1, 167-178.

[14] P. Libermann, Classification and conformal properties of almost Hermitian structures, Differential geometry (Budapest, 1979), 371-391, Colloq. Math. Soc. Jnos Bolyai, 31, North-Holland, Amsterdam-New York, 1982.

[15] S. P. Novikov, Topological invariance of rational classes of Pontrjagin, Dokl. Akad. Nauk SSSR 163 (1965), 298-300.

[16] K. Sekigawa, On some 4-dimensional compact almost Hermitian manifolds, J. Ramanujan Math. Soc. 2 (1987), no. 2, 101-116.

[17] F. Tricerri and L. Vanhecke, Curvature tensors on almost Hermitian manifolds, Trans. Amer. Math. Soc. 267 (1981), no. 2, 365-397.

[18] W. T. Wu, Sur la structure presque complexe d'une variété différentiable réelle de dimension 4, C. R. Acad. Sci. Paris 227 (1948), 1076-1078.

[19] K. Yano, Differential Geometry on Complex and Almost Complex Spaces, Pergamon Press, New York, 1965.

JunGCHAN LEE

Department of Mathematics

SUNGKYUNKWAN UNIVERSITY

SUWON 440-746, KoreA

E-mail address: hanada14@skku.edu

JeongHyeong Park

Department of Mathematics

SUNGKYUNKWAN UNIVERSITY

Suwon 440-746, KoreA

E-mail address: parkj@skku.edu

Kouei Sekigawa

Department of Mathematics

FaCUlty of SCIENCE

NiIgATA University

NiIgATA, 950-2181, JAPAN

E-mail address: sekigawa@math.sc.niigata-u.ac.jp 